\title{
Toxicity and Efficacy Evaluation of an Adeno-Associated Virus Vector Expressing Codon-Optimized RPGR Delivered by Subretinal Injection in a Canine Model of X-linked Retinitis Pigmentosa
}

\author{
Valérie L. Dufour, ${ }^{1}$ Artur V. Cideciyan, ${ }^{2}$ Guo-jie Ye, ${ }^{3}$ Chunjuan Song, ${ }^{3}$ Adrian Timmers, ${ }^{3}$ Perry L. Habecker, ${ }^{4}$ \\ Wei Pan, ${ }^{2}$ Nicole M. Weinstein, ${ }^{5}$ Malgorzata Swider, ${ }^{2}$ Amy C. Durham, Gui-Shuang Ying, ${ }^{2}$ \\ Paulette M. Robinson, ${ }^{3}$ Samuel G. Jacobson, ${ }^{2}$ David R. Knop, Jeffrey D. Chulay, Mark S. Shearman, ${ }^{3}{ }^{3}$ \\ Gustavo D. Aguirre, and William A. Beltran ${ }^{1, *}$ \\ ${ }^{1}$ Division of Experimental Retinal Therapies, Department of Clinical Sciences \& Advanced Medicine, School of Veterinary Medicine, University of Pennsylvania, \\ Philadelphia, Pennsylvania; ${ }^{2}$ Department of Ophthalmology, Scheie Eye Institute, Perelman School of Medicine, University of Pennsylvania, Philadelphia, \\ Pennsylvania; ${ }^{3}$ Applied Genetic Technologies Corporation, Alachua, Florida; ${ }^{4}$ Department of Pathobiology, New Bolton Center, School of Veterinary Medicine, \\ University of Pennsylvania, Kennett Square, Pennsylvania; ${ }^{5}$ Department of Pathobiology, School of Veterinary Medicine, University of Pennsylvania, Philadelphia, \\ Pennsylvania.
}

Applied Genetic Technologies Corporation (AGTC) is developing a recombinant adeno-associated virus (rAAV) vector AGTC-501, also designated rAAV2tYF-GRK1-hRPGRco, to treat X-linked retinitis pigmentosa (XLRP) in patients with mutations in the retinitis pigmentosa GTPase regulator $(R P G R)$ gene. The vector contains a codon-optimized human RPGR cDNA ( $h R P G R c o$ ) driven by a photoreceptor-specific promoter (G protein-coupled receptor kinase 1 [GRK1]), and is packaged in an AAV2 capsid variant with three surface tyrosine residues changed to phenylalanine (AAV2tYF). We conducted a toxicity and efficacy study of this vector administered by subretinal injection in the naturally occurring $R P G R$ mutant (X-linked progressive retinal atrophy 2 [XLPRA2]) dog model. Sixteen RPGR mutant dogs divided into four groups of three to five animals each received either a subretinal injection of $0.07 \mathrm{~mL}$ of AGTC-501 at low $\left(1.2 \times 10^{11}\right.$ vector genome $[\mathrm{vg}] / \mathrm{mL})$, mid $\left(6 \times 10^{11} \mathrm{vg} / \mathrm{mL}\right)$, or high dose $\left(3 \times 10^{12} \mathrm{vg} / \mathrm{mL}\right)$, or of vehicle control in the right eye at early-stage disease. The left eye remained untreated. Subretinal injections were well tolerated and were not associated with systemic toxicity. Electroretinography, in vivo retinal imaging, and histological analysis showed rescue of photoreceptor function and structure in the absence of ocular toxicity in the low- and mid-dose treatment groups when compared with the vehicle-treated group. The high-dose group showed evidence of both photoreceptor rescue and posterior segment toxicity. These results support the use of AGTC-501 in clinical studies with patients affected with XLRP caused by $R P G R$ mutations and define the no-observed-adverse-effect level at $6 \times 10^{11} \mathrm{vg} / \mathrm{mL}$.

Keywords: XLRP, retinal degeneration, gene therapy, $R P G R$

\section{INTRODUCTION}

X-LINKED RETINITIS PIGMENTOSA (XLRP) accounts for 6$20 \%$ of all cases of $\mathrm{RP}^{1-3}$ and $>350$ different mutations (http://rpgr.hgu.mrc.ac.uk) in the retinitis pigmentosa GTPase regulator $(R P G R)$ gene are found in $>70 \%$ of families affected by XLRP and $\sim 15 \%$ of simplex RP cases. $^{4-8} R P G R$-XLRP often shows greater severity and earlier onset than other forms of RP. Affected male patients typically exhibit night blindness during childhood and progressive visual field constriction that leads to legal blindness in their fourth decade of life; yet, a spectrum of severity and phenotypes have been observed in families carrying different $R P G R$ mutations. ${ }^{9}$

Among the several alternative splice variants identified, the two most abundantly expressed RPGR isoforms in the retina are constitutive $\mathrm{RPGR}^{\mathrm{ex1} 1-19}$ and $\mathrm{RPGR}^{\mathrm{ex} 1-\mathrm{ORF} 15} \cdot{ }^{10-12}$ Both transcripts are localized in the connecting cilia of rod and cone photoreceptor cells. ${ }^{13}$ RPGR $^{\text {ex1-19 }}$ is thought to play a role in photoreceptor development, while RPGR ${ }^{\text {ex1-ORF15 }}$ is considered to be critical at

${ }^{*}$ Correspondence: Dr. William A. Beltran, Division of Experimental Retinal Therapies, Department of Clinical Sciences \& Advanced Medicine, School of Veterinary Medicine, University of Pennsylvania, 3900 Delancey Street, Philadelphia, PA 19104. E-mail: wbeltran@vet.upenn.edu; Dr. Mark S. Shearman, Applied Genetic Technologies Corporation, 14193 NW 119th Terrace, Suite 10, Alachua, FL 32615. E-mail: mshearman@agtc.com 
regulating protein trafficking between inner and outer segments and maintains the structural and functional integrity of mature photoreceptors. ${ }^{13-16}$

The use of two naturally occurring canine models of $R P G R$-XLRP ${ }^{17-19}$ provided the first proof of concept that an AAV-mediated RPGR gene augmentation strategy could prevent disease onset and arrest its progression when delivered subretinally at early-stage disease. ${ }^{20}$ Long-term follow-up of treatment for the early and aggressive forms of $\mathrm{X}$-linked retinal degeneration in dogs, $\mathrm{X}$-linked progressive retinal atrophy 2 (XLPRA2), by gene augmentation delivered at early-, mid-, and late-stage disease showed sustained preservation of retinal structure and function, and retained visually guided behavior for $>2$ years. ${ }^{21} \mathrm{~A}$ beneficial effect of $R P G R$ gene augmentation targeted to rods and cones was also found in rodent models. ${ }^{22,23}$

To treat patients affected with RPGR-XLRP, Applied Genetic Technologies Corporation (AGTC) is developing a recombinant adeno-associated virus (rAAV) viral vector (rAAV2tYF-GRK1-hRPGRco or AGTC-501), which consists of a codon-optimized human RPGR ${ }^{\text {ex1-ORF15 }}$ cDNA driven by the photoreceptor-specific $\mathrm{G}$ protein-coupled receptor kinase 1 (GRK1) promoter, and packaged in an AAV2 capsid variant with three tyrosine to phenylalanine mutations on its surface (AAV2tYF). This vector was previously validated in short-term proof-of-concept studies in $R P G R$-mutant (XLPRA2) dogs, ${ }^{24}$ and its toxicity and efficacy were evaluated in RPGR-deficient Rd9 mice. ${ }^{25}$

As part of the efforts to develop a product candidate for treatment of humans with XLRP caused by mutation in the $R P G R$ gene, an Investigational New Drug (IND)-enabling toxicity and efficacy study of AGTC-501 was conducted in XLPRA2 dogs.

\section{MATERIALS AND METHODS Vector production}

The vector AGTC-501 was produced using a recombinant herpes simplex virus (rHSV)-assisted vector expansion (HAVE) system in suspension-cultured baby hamster kidney (sBHK) cells, and followed a similar procedure to that used in two previous studies. ${ }^{26,27}$ Two rHSV helper viruses, one containing the AAV2 rep and AAV2tYF cap genes and the other containing the codon-optimized human RPGR cDNA (hRPGRco) expression cassette, were used to coinfect sBHK cells grown in serum-free medium. One day later, the cells were lysed with Triton X-100 detergent, treated with Benzonase (Merck), clarified by filtration, purified by AVB Sepharose (GE Life Sciences) affinity chromatography followed by CIM SO3- (BIA separations) cation-exchange chromatography, and eluted in $2.6 \times$ balanced salt solution containing $0.014 \%$ (v/v) Tween 20 (BSST). The purified bulk was concentrated and buffer exchanged to $1 \times$ BSST (drug substance) and sterile filtered $(0.2 \mu \mathrm{m})$ to generate drug product and stored at $<65^{\circ} \mathrm{C} .{ }^{28-30}$

\section{Vector characterization}

Vector concentration (vector genomes [vg] per $\mathrm{mL}$ ), vector infectivity (tissue culture 50\% infectious dose), purity (silver-stained sodium dodecyl sulfate-polyacrylamide gel electrophoresis analysis), and concentrations of endotoxin and HSV protein were measured as previously described. ${ }^{31}$ Concentrations of the BHK protein, bovine serum albumin, Benzonase, and AVB ligand were measured by enzyme-linked immunosorbent assay using commercially available kits. Concentrations of HSV and BHK DNA were measured by quantitative polymerase chain reaction (qPCR). Testing for mycoplasma, bacteria, and fungi was performed using standard microbiological methods. Testing for infectious HSV was performed by serial passage in V27 cells.

\section{Subretinal injections}

Studies were carried out in strict accordance with the recommendations in the Guide for the Care and Use of Laboratory Animals of the National Institutes of Health, in compliance with the USDA's Animal Welfare Act, Animal Welfare Regulations, and the ARVO Statement for the Use of Animals in Ophthalmic and Vision Research. The protocols were approved by the Institutional Animal Care and Use Committee of the University of Pennsylvania (IACUC\# 803269).

The dogs were bred and maintained at the University of Pennsylvania Retinal Disease Studies Facility (RDSF; Table 1). For identification purpose, all dogs used in this study were microchipped and tattooed. The study was designed in a manner that addressed the key components of the principles of Good Laboratory Practice (GLP) regulations as set forth in 21 CFR Part 58, GLP for Nonclinical Laboratory Studies, but was not formally fully GLP compliant. This included the involvement of quality control and quality assurance units throughout the duration of the study.

The study was designed to evaluate the toxicity and efficacy of AGTC-501 in RPGR mutant dogs when administered by subretinal injection of $0.07 \mathrm{~mL}$ at three different doses of viral solution in the right eye (low dose: $1.2 \times 10^{11} \mathrm{vg} / \mathrm{mL}$ in four eyes, mid dose: $6.0 \times 10^{11} \mathrm{vg} / \mathrm{mL}$ in four eyes, or high dose: $3.0 \times 10^{12} \mathrm{vg} / \mathrm{mL}$ in five eyes), or of the vehicle control (Alcon BSS with $0.014 \%$ Tween-20 in three eyes). The contralateral left eyes remained untreated. Subretinal injections were administered as previously reported using a refurbished device with a 39-gauge polyimide subretinal cannula (Retinaject; Surmodics, Inc., Eden Prairie, MN) ${ }^{32,33}$ An injection was considered successful if the viral solution was injected into the subretinal space at the first attempt, resulting in a bleb that could be immediately visualized and documented by fundus photography (Retcam shuttle; Natus Medical, Inc., Pleasanton, CA). A total of 16 dogs ranging from 5 to 6.5 weeks of age were enrolled for the entire duration of the study. 
All dosing of animals as well as in-life and postlife assessments were performed in a masked manner with investigators acquiring and analyzing the data as well as study director masked. Study day 0 was the day of injection for each animal. All animals were sacrificed 20-21 weeks postinjection.

\section{Pre-, per-, and postoperative medication regimen}

On the morning of surgery, dogs received oral antibiotic (Clavamox, $12.5-20 \mathrm{mg} / \mathrm{kg}$ ) and corticosteroid (Prednisone, $1 \mathrm{mg} / \mathrm{kg}$ ) medication, together with topical antibiotics, anti-inflammatories, and mydriatics. Immediately after the end of the surgical procedure, a subconjunctival injection of triamcinolone acetonide $(2 \mathrm{mg})$, and topical application of atropine sulfate $(1 \%)$ and antibiotic + steroid (NeoPolyDex) ointment were given in the injected eye only. Topical application of corticosteroid suspension (prednisolone acetate 1\%) was performed twice a day in the treated eye for 2 weeks, and then once daily for the following 2 weeks. Atropine sulfate (1\%) ointment was applied in the treated eye once daily during the first week. Oral administration of antibiotics (Clavamox, $12.5 \mathrm{mg} / \mathrm{kg}$ ) was given twice a day for 6 weeks, oral administration of corticosteroids $(1 \mathrm{mg} / \mathrm{kg})$ was given twice a day for 2 weeks, then once a day for an additional 2 weeks. At 4 weeks postinjection, a second subconjunctival injection of triamcinolone $(4 \mathrm{mg})$ was given in the treated eye under topical anesthesia.

\section{In-life toxicology and efficacy assessment}

Cage-side observation, physical examination, and body weights. Animals were observed daily for signs of morbidity/mortality. A physical examination conducted by a veterinarian was performed before injection, on the day of injection (study day 0 ), on study day 1 , and weekly thereafter ( \pm 3 days). Weights were recorded at each examination. Abnormal findings including ocular signs were recorded.

Ophthalmic examination. A board-certified veterinary ophthalmologist performed in-life ophthalmic examinations that included slit-lamp biomicroscopy, tonometry, and indirect ophthalmoscopy. Inflammatory changes (conjunctival hyperemia, chemosis or discharge, anterior chamber flare/cells and cellularity/precipitates in the vitreous) and changes in transparency of ocular media (cornea/lens/vitreous) were graded as none, mild, moderate, or severe. Intraocular pressure (IOP) measurements were also recorded for each eye. Examinations were conducted before vector administration, 7 days ( \pm 2 days), $2,6,10,15$, and 20-21 weeks ( \pm 3 days) after vector administration, and all abnormalities were documented. Fundus photography of both eyes was performed to document the retinal appearance before vector administration, immediately after the subretinal injection, at 10 weeks ( \pm 3 days) and 20-21 weeks ( \pm 3 days) after vector administration.

For each categorical outcome measure (e.g., mortality, ocular flare), a frequency table was used to check the frequency distribution; proportions were calculated, and compared across all treatment groups and between each active treatment group (low, mid, and high dose) versus the vehicle-control group using Fisher's exact test. Within the same treatment group, McNemar's test was used to compare the proportion between treated eyes and untreated fellow eyes.

For continuous outcome measures (e.g., IOP), descriptive analysis included reporting of mean and standard deviation (SD). The comparison of means across all treatment groups was performed using one-way analysis of variance (ANOVA). Regardless of whether the overall group effect was statistically significant, a pairwise comparison between each active treatment group (low, mid, and high dose) and the vehicle-control group was performed by using the two-sample $t$-test. Within each treatment group, the paired $t$-test was performed to compare the means between treated eyes and untreated fellow eyes. All statistical analyses were performed using SAS v9.4 (SAS Institute, Inc., Cary, NC).

Electroretinography. Recordings were conducted at 10 weeks ( \pm 3 days) and $19-20$ weeks ( \pm 3 days) posttreatment. Pupils were dilated with topical application of atropine sulfate 1\% (Akorn, Inc., Lake Forest, IL), tropicamide $1 \%$ (Akorn, Inc.), and phenylephrine 10\% (Paragon Biotech, Portland, OR). After induction with intravenous propofol (Zoetis, Kalamazoo, MI), dogs were maintained under general inhalation anesthesia (isoflurane 2-3\%; Akorn, Inc.). Full-field flash electroretinography (ERG) was performed on both eyes using a custom-built Ganzfeld dome fitted with LED stimuli from a ColorDome stimulator (Diagnosys LLC, Lowel, MA). After $20 \mathrm{~min}$ of dark adaptation rod and mixed rod-cone-mediated responses to single $4 \mathrm{~ms}$ white flash stimuli of increasing intensities $\left(-3.7\right.$ to $0.5 \mathrm{log}$ cd.s. $\mathrm{m}^{-2}$ ) were recorded. After $5 \mathrm{~min}$ of white light adaptation $\left(10.6 \mathrm{~cd} . \mathrm{m}^{-2}\right)$, conemediated responses to a series of single white flashes $(-2.7$ to $0.5 \mathrm{log}$ cd.s. $\mathrm{m}^{-2}$ ) and to $29.4 \mathrm{~Hz}$ flicker stimuli $(-2.7$ to $0.2 \log$ cd.s. $\mathrm{m}^{-2}$ ) were recorded. Amplitudes of the b-wave of the dark-adapted rod response, a- and b-waves of the dark-adapted rod-cone mixed response, and the peak of amplitudes of the light-adapted cone single flash and $29.4 \mathrm{~Hz}$ flicker were measured.

For these continuous outcome measures (amplitudes of ERG waves), descriptive analysis included reporting of mean and SD. To account for potential animal-to-animal variability of ERG amplitudes, the mean difference between the injected right (OD) and contralateral uninjected left (OS) eyes was calculated for each of the three groups treated with the test article and compared with 
that of the vehicle-control group. The comparison of means across all treatment groups was performed using one-way ANOVA. Regardless of whether the overall group effect was statistically significant, a pairwise comparison between each active treatment group (low, mid, and high dose) and the vehicle-control group was performed by using the two-sample $t$-test. Within each treatment group, the paired $t$-test was used to compare the means between treated eyes and their untreated fellow eyes. All statistical analyses were performed using SAS v9.4 (SAS Institute, Inc.).

Confocal laser ophthalmoscopy/optical coherence tomography retinal imaging. Imaging sessions were conducted at 10 weeks ( \pm 3 days) and 19-20 weeks ( \pm 3 days) post-treatment. En-face confocal laser ophthalmoscopy (cSLO) and retinal cross-sectional optical coherence tomography (OCT) imaging were performed with the animal under general anesthesia using isoflurane (isoflurane 2$3 \%$ ). Overlapping en-face images of reflectivity with nearinfrared illumination $(820 \mathrm{~nm})$ were obtained (Spectralis HRA+OCT; Heidelberg, Heidelberg, Germany) with a $55^{\circ}$ diameter lens to delineate fundus features such as optic nerve, retinal blood vessels, boundaries of injection blebs, retinotomy sites, and other local changes. Shortwavelength $(488 \mathrm{~nm})$ autofluorescence and reflectance imaging were used to outline the boundaries of the tapetum and the RPE. Spectral-domain OCT was performed with linear and raster scans using the $30^{\circ}$ lens. Overlapping $\left(30^{\circ} \times 20^{\circ}\right)$ raster scans were recorded covering large regions of the retina $\left(\sim 100^{\circ}\right.$ wide) that included in injected eyes, both treated and untreated areas.

Postacquisition analyses of OCT data collected at week 10 included selection of a single OCT B-scan (spanning $30^{\circ}$ in length) located within the central region of the bleb/ treated area in the injected (OD) eyes, and another OCT Bscan located in the bleb-equivalent area of the contralateral (OS) uninjected eye. Manual segmentation of the ONL was performed using the Spectralis Eye Explorer program. ONL thickness was measured along each single OCT B scan at $15-17$ locations evenly spaced (every $1.76^{\circ}$ ) unless the location fell at the level of retinal blood vessel. The mean $( \pm \mathrm{SD})$ value of ONL thickness was calculated for each OCT B scan.

Postacquisition analyses of OCT data collected at weeks 19-20 were more extensive and included generation of ultra-wide-angle composite images from the infrared images, registration of each of $\sim 1,500$ to $1,800 \mathrm{~B}$-scans to their corresponding retinal location across each retina, resampling of the registered OCT data into regularly spaced bins of a coordinate system centered on the optic nerve. ${ }^{24}$ All longitudinal reflectivity profiles collected in each bin were aligned and averaged before performing manual segmentation of the two boundaries that define ONL thickness. ONL thicknesses were assigned to a pseudocolor scale to generate topographic images. Injection blebs from the postsurgery images, location of the fovea-like area, and the boundary of the tapetum were overlaid on resulting ONL maps. ONL results within the injection bleb were sampled and compared with results from anon-bleb control area of the same eye (OD), as well as from the blebequivalent area of the contralateral uninjected eye (OS).

For these continuous outcome measures (ONL thickness by OCT), descriptive analysis included reporting of mean and SD. To account for potential animal-to-animal variability of ONL thickness, the mean difference between the injected (OD) and uninjected (OS) eyes was calculated for each of the three groups treated with the test article and compared with that of the vehicle-control group. The comparison of means across all treatment groups was performed using one-way ANOVA. Regardless of whether the overall group effect was statistically significant, a pairwise comparison between each active treatment group (low, mid, and high dose) and the vehicle-control group was performed by using the two-sample $t$-test. Within each treatment group, the paired $t$-test was performed to compare the means between treated eyes and their untreated fellow eyes. All statistical analyses were performed using SAS v9.4 (SAS Institute, Inc.).

Immunological studies. Serum was obtained before treatment, at 10 weeks ( \pm 3 days) and 20-21 weeks ( \pm 3 days) after treatment. Antibodies to AAV capsid were measured using a neutralization assay, as previously described. ${ }^{34}$ Antibodies to hRPGR protein were measured using an enzyme-linked immunosorbent assay developed to detect anti-hRPGR antibodies in dog serum. In brief, 96-well microtiter plates were coated with recombinant human RPGR protein or with purified dog immunoglobulin $\mathrm{G}$ ( $\operatorname{IgG}$ ). After incubation, dog sera (test samples and negative controls) or serial dilutions of mouse antihRPGR reference standard and positive controls were added to the wells coated with hRPGR antigen. Blocking buffer was added to the selected wells coated with dog IgG. Antibodies in the sera that bind to the hRPGR capture antigen were then detected using horseradish peroxidase (HRP)-labeled secondary antibody cocktail (goat antimouse IgG and goat antidog $\mathrm{IgG}$ ), followed by addition of an enzyme substrate (TMB) that changes color in the presence of HRP. Serum antibody titers were quantified by comparison with the standard curve generated from dilutions of the reference standard and expressed as inverse of greatest dilution.

Clinical pathology. Blood for hematology (complete blood count), routine coagulation profile, and clinical chemistry was collected before vector administration and at 2 weeks ( \pm 3 days) and 20-21 weeks ( \pm 3 days) after treatment. Animals were fasted overnight before collection. Analysis was performed at the University of 
Pennsylvania School of Veterinary Medicine clinical pathology laboratory, and results were reviewed and interpreted by a board-certified veterinary clinical pathologist.

For these continuous outcome measures (clinical pathology values), descriptive analysis was performed using mean and SD. The comparison of means across all treatment groups was performed using one-way ANOVA. Regardless of whether the overall group effect was statistically significant, a pairwise comparison between each active treatment group (low, mid, and high dose) and the vehicle-control group was performed by using the twosample $t$-test. Within each treatment group, the paired $t$-test was used to compare the means between treated eyes and their untreated fellow eyes. All statistical analyses were performed using SAS v9.4 (SAS Institute, Inc.).

\section{Postmortem toxicology and efficacy assessment}

Necropsy. Animals were sacrificed $\sim 20-21$ weeks ( \pm 3 days) after treatment. At the time of scheduled sacrifice, animals were exsanguinated under general anesthesia induced with a low dose of commercial pentobarbitalbased euthanasia solution (Euthasol; Virbac USA) that was administered through intravenous injection. Euthanasia was then performed by injection of an overdose of the commercial pentobarbital-based euthanasia solution once the exsanguination process was completed. A thorough external and internal examination including body orifices, cranial, thoracic, abdominal organs, and tissues was performed by a board-certified veterinary pathologist. Using ultraclean techniques, tissues designated for qPCR analyses other than the brain were collected immediately after death and frozen in liquid nitrogen. Brain, heart, lung (pair), liver and gallbladder, kidneys (pair), spleen, thymus, adrenals (pair), uterus, ovaries or testes (pair) were weighed. After enucleation, a blue tissue marking dye was used to stain the sclera in the quadrant that included the prior subretinal bleb based on injection records of its location. The ocular globes including a short piece of optic nerve were fixed in alcoholic Bouin's for $72 \mathrm{~h}$ before transfer into $70 \%$ ethanol. The orientation of the trimming was adjusted, so that paraffin-embedded sections of the injected eyes would include the optic nerve head and extend through both the untreated inferotemporal and the treated superonasal areas of injected eyes. The contralateral uninjected eyes were oriented and trimmed in the same manner, and tissue sections from both eyes were stained with hematoxylin and eosin (H\&E).

Using extraclean procedures to prevent crosscontamination (clean gloves, scissors, and forceps for each tissue collected), samples from the following tissues were collected and stored at $-80^{\circ} \mathrm{C}$ for biodistribution analysis in the future if needed: liver, spleen, heart, lung (pair), kidneys (pair), parotid gland, ovaries (pair), and testes (pair), and gross lesions. Samples of the same tissues were also collected for histology and fixed in $10 \%$ neutral buffered formalin.

The brain was removed at the end of the necropsy and weighed. The optic chiasma was collected for histopathology, then the brain was sectioned sagittally and placed in $10 \%$ neutral buffered formalin. The following regions of the brain were dissected to obtain samples for histopathology and qPCR analysis after 48, but no longer than $72 \mathrm{~h}$ in formalin: optic tract (right and left), lateral geniculate nucleus (right and left), occipital cortex (right and left), and cerebellum (right and left). Samples collected for qPCR analyses were transferred to $70 \%$ ethanol and stored. As previous biodistribution studies of rAAV2tYF after subretinal delivery had already been performed, ${ }^{26,30}$ biodistribution analysis on the collected samples was not performed, yet the tissues were collected in case of future need or request from the US Food and Drug Administration (FDA).

Group means and SD were calculated for terminal body weight immediately postexanguination, organ weight, organ-to-body weight ratios, organ-to-brain weight ratios. The comparison of means across all treatment groups was performed using one-way ANOVA. Regardless of the statistical significance of the overall group effect, a pairwise comparison between each active treatment group (low, mid, and high dose) and the vehicle-control group was performed by using the two-sample $t$-test. Within each treatment group, the paired $t$-test was performed to compare the means between treated eyes and their untreated fellow eyes. All statistical analyses were performed using SAS v9.4 (SAS Institute, Inc.).

Histopathology and ocular histopathology. Two H\&E-stained slides of each tissue were examined by a board-certified veterinary pathologist from the School of Veterinary Medicine, University of Pennsylvania, and independently peer reviewed by a third party's pathologist (Biogen, Cambridge, MA) who was masked to the treatment groups. H\&E-stained paraffin sections of the ocular globes that included the optic nerve head and the bleb/ treated and bleb-equivalent area in contralateral uninjected eyes were digitally scanned (Leica Aperio Versa 200; Leica, Buffalo Grove, IL).

Manual measurement of the ONL thickness was performed using the Aperio ImageScope (Leica) software at $1 \mathrm{~mm}$ intervals extending from the edge of the optic nerve head to the ora serrata. Spider graphs of ONL thickness were constructed for both eyes as previously reported. ${ }^{35}$ Based on the location of the bleb, the mean ONL thickness taken from three to four locations within the bleb/ treated area was calculated. Similarly, three to four blebequivalent locations were selected in the contralateral uninjected eyes to calculate the mean ONL thickness in these eyes. To account for potential animal-to-animal 
variability of ONL thickness, the mean difference between the injected (OD) and uninjected (OS) eyes was calculated for each of the three groups treated with the test article and compared with that of the vehicle-control group.

The comparison of means across all treatment groups was performed using one-way ANOVA. Regardless of whether the overall group effect was statistically significant, a pairwise comparison between each active treatment group (low, mid, and high dose) and the vehicle-control group was performed by using the two-sample $t$-test. Within each treatment group, the paired $t$-test was used to compare the means between treated eyes and their untreated fellow eyes. All statistical analyses were performed using SAS v9.4 (SAS Institute, Inc.).

\section{RESULTS AND DISCUSSION}

\section{Relevance for clinical trials}

Results of this study supported a clinical trial in which individuals with XLRP due to RPGR-ORF 15 mutation are receiving AGTC-501 administered by subretinal injection. The clinical trial is a dose-escalation study in which successive groups of subjects receive progressively increasing dose of study agent. Details are available at https:// clinicaltrials.gov/ct2/show/NCT03316560.

\section{Objective and study design}

The study was designed to evaluate the efficacy and toxicity of rAAV2tYF-GRK1-hRPGRco (AGTC-501) administered by subretinal injection in XLPRA2 dogs, a naturally occurring canine model of early-onset and severe XLRP that carries a frameshift mutation in exonORF15 of $R P G R .^{18,19}$ This IND-enabling study conducted by the Division of Experimental Retinal Therapies (ExpeRTs) at the School of Veterinary Medicine, University of Pennsylvania, was carried out in strict accordance with the recommendations in the Guide for the Care and Use of Laboratory Animals of the National Institutes of Health in compliance with the USDA's Animal Welfare Act, Animal Welfare Regulations, and the ARVO Statement for the Use of Animals in Ophthalmic and Vision Research. The protocols were approved by the Institutional Animal Care and Use Committee of the University of Pennsylvania.

A total of 16 XLPRA2 dogs (age: 5-6.5 weeks), including hemizygous males and homozygous females, were randomly allocated to one of four treatment groups. In one dog from the high-dose group (Animal ID Z575), the eccentric location of the subretinal bleb and its rapid flattening in the immediate postop period (Fig. 1D1) led us to add a fifth animal to this treatment group and include three dogs in the vehicle-treated group. Four animals were allocated to the other two groups (see study design summarized in Table 1). These dogs were retained in the study and followed for 20-21 weeks post-treatment, at which point they were euthanatized for histopathological analysis.

Each dog received a $0.07 \mathrm{~mL}$ subretinal injection of either vehicle (Alcon BSS with 0.014\% Tween-20) or one of three doses of the test article (AGTC-501) in the right eye (OD). Animals in the low-dose group received the test article (AGTC-501) at $1.2 \times 10^{11} \mathrm{vg} / \mathrm{mL}$, animals in the middose group at $6.0 \times 10^{11} \mathrm{vg} / \mathrm{mL}$ (mid dose), and animals in the high-dose group at $3.0 \times 10^{12} \mathrm{vg} / \mathrm{mL}$. The left eye (OS) remained untreated. At the time of surgery, visualization through the operating microscope of the formation of a large subretinal bleb proportional to the injected volume (Fig. 1A1-D1) immediately after the injection was the criteria for retaining dogs in the study.
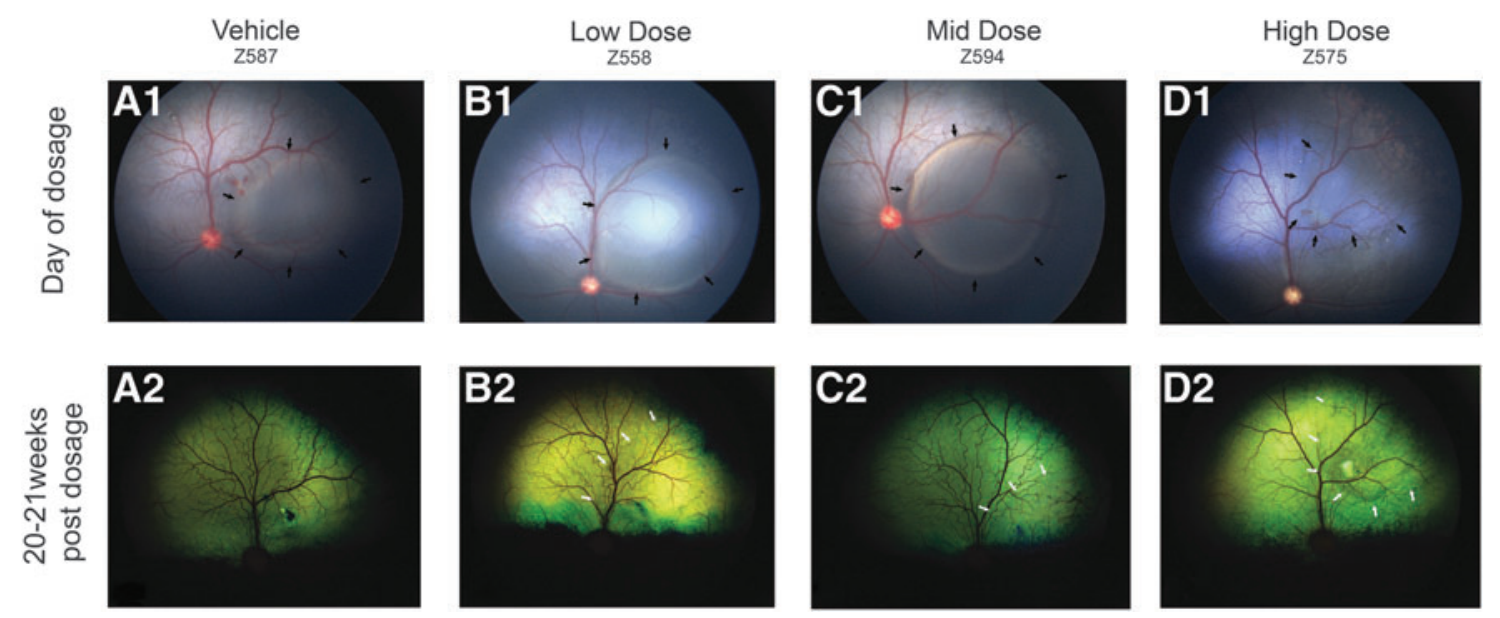

Figure 1. Fundus photographs from selected dogs of all treatment groups illustrating the location and appearance of the treated retinal area. $\left(\mathbf{A}_{\mathbf{1}}-\mathbf{D}_{\mathbf{1}}\right)$ Subretinal blebs (border between attached and detached retina delimited by black arrows) immediately after subretinal vector administration. $\left(\mathbf{A}_{\mathbf{2}}-\mathbf{D}_{\mathbf{2}}\right.$ ) Fundus appearance 20-21 weeks post-treatment. A border (white arrows) delineating the retinal-treated area can be seen in eyes that were treated with low dose $\left(1.2 \times 10^{11} \mathrm{vg} / \mathrm{mL}\right)$, mid dose $\left(6.0 \times 10^{11} \mathrm{vg} / \mathrm{mL}\right)$, and high dose $\left(3.0 \times 10^{12} \mathrm{vg} / \mathrm{eye}\right)$ of test article AGTC-501. AGTC, Applied Genetic Technologies Corporation; vg, vector genome. 
Table 1. Study design to evaluate subretinal injection of AGTC-501 in RPGR mutant dogs

\begin{tabular}{|c|c|c|c|c|c|}
\hline \multirow[b]{2}{*}{ Group } & \multirow[b]{2}{*}{$\begin{array}{l}\text { No. of } \\
\text { Animals }\end{array}$} & \multirow[b]{2}{*}{ Dog ID and Sex } & \multicolumn{3}{|c|}{ Dosage Level } \\
\hline & & & $\begin{array}{c}\text { Vector } \\
\text { Concentration } \\
(\mathrm{vg} / \mathrm{mL})\end{array}$ & $\begin{array}{l}\text { Injection } \\
\text { Volume } \\
\text { (mL) }\end{array}$ & $\begin{array}{c}\text { Total } \\
\text { Dose } \\
\text { (vg/eye) }\end{array}$ \\
\hline Vehicle & 3 & $\begin{array}{l}\text { Z559 F, Z569 M, } \\
\text { Z587 M }\end{array}$ & 0 (control) & 0.07 & 0 \\
\hline Low dose & 4 & $\begin{array}{l}\text { Z558 F, Z566 M, } \\
\text { Z576 M, Z605 M }\end{array}$ & $1.2 \times 10^{11}$ & 0.07 & $8.4 \times 10^{9}$ \\
\hline Mid dose & 4 & $\begin{array}{l}\text { Z555 M, Z574 M, } \\
\text { Z594 M, Z603 M }\end{array}$ & $6.0 \times 10^{11}$ & 0.07 & $42 \times 10^{9}$ \\
\hline High dose & 5 & $\begin{array}{l}\text { Z570 M, Z575 M, } \\
\text { Z592 M, Z596 M, } \\
\text { Z604 M }\end{array}$ & $30 \times 10^{11}$ & 0.07 & $210 \times 10^{9}$ \\
\hline
\end{tabular}

Each animal received a subretinal injection in the right eye. The left eye was left untreated and used as control.

$R P G R$, retinitis pigmentosa GTPase regulator.

Physical examination and body weights were conducted before dosage, on day of dosage (day 0), on study day 1 , and weekly thereafter. Clinical pathology on blood samples was performed prior dosage, on weeks 2 and 2021. Serum for immunological studies was collected prior treatment, and on weeks 10 and 20-21. Ophthalmic examination (slit-lamp biomicroscopy, indirect ophthalmoscopy, and tonometry) was done prior treatment and on weeks 1, 2, 6, 10, 15, and 20-21. Fundus photography was performed prior dosage, on day of dosage to document the bleb localization, on week 10 , and before termination (week 20-21). Noninvasive retinal imaging also included cSLO and spectral-domain OCT that were conducted on weeks 10 and 19-20. Functional retinal assessment by ERG was conducted on weeks 10 and 19-20. A full body necropsy and histopathological analysis were performed at termination on all study animals (Table 2).

\section{Summary of data}

Clinical observations. There were no test-articlerelated abnormalities. All animals reached the end of the study (week 20-21) with good body condition. One dog from the vehicle-treatment group, one dog from the lowdose treatment group, and one dog from the mid-dose treatment group presented cutaneous signs of demodicosis for the last 2-4 weeks of the study. This is a common finding in young dogs, especially when treated with high dose of systemic corticosteroids. One dog from the high-dosetreated group presented a prolapse of the nictitans gland in the uninjected eye at week 2 that persisted until the end of the study. This sporadic clinical finding in dogs is observed occasionally in our colony. ${ }^{36}$ One dog from the mid-dose treatment group presented at week 10 a severe bilateral blepharitis and adenomegaly of both submandibular lymph nodes. The condition was treated with topical and systemic antibiotics and corticosteroid regimen, and was controlled by week 14 . Moderate signs of blepharitis and adenomegaly recurred on week 19 but were left untreated. Histopathology examination conducted 3 days later established a diagnosis of juvenile sterile

Table 2. Summary of assessment procedures

\begin{tabular}{|c|c|c|c|c|c|c|c|c|c|}
\hline & $\begin{array}{l}\text { Subretinal } \\
\text { Injection }\end{array}$ & $\begin{array}{c}\text { Physical Examination + } \\
\text { Body Weight }\end{array}$ & $\begin{array}{l}\text { Clinical } \\
\text { Pathology }\end{array}$ & $\begin{array}{l}\text { Titration } \\
\text { of } \mathrm{Nab}\end{array}$ & $\begin{array}{l}\text { Eye Examination + } \\
\qquad I 0 P\end{array}$ & $\begin{array}{c}\text { Fundus } \\
\text { Photography }\end{array}$ & $\begin{array}{c}\text { cSLO+ } \\
\text { OCT }\end{array}$ & $E R G$ & $\begin{array}{l}\text { Termination } \\
\text { and Necropsy }\end{array}$ \\
\hline Predosage & & $x$ & $x$ & $x$ & $x$ & $x$ & $x$ & & \\
\hline Day of dosage & $X$ & $x$ & & & & $x$ & & & \\
\hline Day 1 & & $x$ & & & & & & & \\
\hline Week 1 & & $x$ & & & $x$ & & & & \\
\hline Week 2 & & $x$ & $X$ & & $x$ & & & & \\
\hline Week 3 & & $x$ & & & & & & & \\
\hline Week 4 & & $x$ & & & & & & & \\
\hline Week 5 & & $x$ & & & & & & & \\
\hline Week 6 & & $x$ & & & $x$ & & & & \\
\hline Week 7 & & $x$ & & & & & & & \\
\hline Week 8 & & $x$ & & & & & & & \\
\hline Week 9 & & $x$ & & & & & & & \\
\hline Week 10 & & $x$ & & $X$ & $X$ & $X$ & $x$ & $X$ & \\
\hline Week 11 & & $x$ & & & & & & & \\
\hline Week 12 & & $x$ & & & & & & & \\
\hline Week 13 & & $x$ & & & & & & & \\
\hline Week 14 & & $x$ & & & & & & & \\
\hline Week 15 & & $x$ & & & $X$ & & & & \\
\hline Week 16 & & $x$ & & & & & & & \\
\hline Week 17 & & $x$ & & & & & & & \\
\hline Week 18 & & $x$ & & & & & & & \\
\hline Week 19 & & $x$ & & & & & $x$ & $x$ & \\
\hline Week 20 & & $x$ & $X$ & $X$ & $X$ & $X$ & & & $x$ \\
\hline
\end{tabular}

$\mathrm{X}$ indicates procedure performed, gray shading indicates procedure not performed.

cSLO + OCT, confocal laser ophthalmoscopy + spectral-domain optical coherence tomography; ERG, electroretinography; IOP, intraocular pressure; Nab, neutralizing antibodies. 
granulomatous dermatitis ${ }^{37}$ and lymphadenitis. This is a rare immune-mediated condition that affects young dogs and that is well controlled by corticosteroids therapy. Thus, it was not considered to be test article (AGTC-501) related. In summary, administration of AGTC-501 by subretinal injection was well tolerated, and not associated with any clinical signs of systemic toxicity.

Ophthalmic examinations. Ophthalmic examinations were unremarkable in both eyes prior dosage and in the untreated eyes throughout the duration of the study. Multifocal areas of retinal separation were found at week 20 in the tapetal region of both eyes in one high-dose dog (Animal ID Z596). Focal and transient retinal separations have been observed occasionally by the authors in untreated $R P G R$ mutant dogs at similar ages (unpublished data). The cause and mechanism of these lesions are currently unknown.

Ophthalmic findings in the three eyes that were injected with vehicle are summarized in Supplementary Table S1. All eyes showed the characteristic focal pigmentation at the retinotomy site, and a bleb border suggestive of a rescue effect was not observed within the treated area (Fig. 1A2). One animal developed a retinal fold from week 1 to 6 , a transient developmental retinal defect that is commonly seen in young dogs. This animal also had a focal area of dark blue or brown tapetal color change close to the treated region from week 1 to 20 (Supplementary Fig. S1A for illustration). This tapetal alteration is suspected to be a light-induced injury of the tapetum caused at the time of injection by illumination from the operating microscope, OCT examination of this region showed no structural abnormalities of the neuroretina (Supplementary Fig. S1B for illustration).

Ophthalmic findings in the four eyes that were injected with AGTC-501 at $1.2 \times 10^{11} \mathrm{vg} / \mathrm{mL}$ (low dose) are summarized in Supplementary Table S2. One animal presented an area of retinal atrophy associated with the injection site at weeks 10 and 20 (identified by OCT) that was most likely surgically induced. A subtle bleb border suggestive of retinal thickness preservation within the treated area was visible in three of four animals at week 15 and/or 20 (Fig. 1B2).

Ophthalmic findings in the four eyes that were injected with AGTC-501 at $6.0 \times 10^{11} \mathrm{vg} / \mathrm{mL}$ (mid dose) are summarized in Supplementary Table S3. All four eyes had focal pigmentation at the retinotomy site, and three of four eyes also had dark blue tapetal color change outside of the treated area. One dog developed a surgically induced preretinal hemorrhage that became a vitreal clot at week 1 and resolved by week 2 . The same animal developed an incipient cataract along the posterior Y sutures that started at week 10 and persisted until the end of the study that did not impair retinal imaging, and was considered as an incidental finding. In three of the four eyes, a subtle bleb border suggestive of retinal thickness preservation within the treated area was visible as early as week 6 (Fig. 1C2).
Ophthalmic findings in the five eyes that were injected with AGTC-501 at $3.0 \times 10^{12} \mathrm{vg} / \mathrm{mL}$ (high dose) are summarized in Supplementary Table S4. At week 10, two eyes showed fine punctate crystalline corneal epithelial/ subepithelial deposits commonly seen in dogs after chronic topical steroid administration. A bleb border suggestive of retinal preservation within the treated area was visible as early as week 6 and found in four of five eyes by week 10 (Fig. 1D2). At week 10, one dog showed dilated retinal vessels (Animal ID Z575) that resolved by week 15. At the same time point, one dog (Animal ID Z592) showed some vitreal cells, changes in vitreous transparency, and signs of chorioretinitis with dilated retinal vessels that remained until week 15. At week 20, the retinal changes within the treated area progressed to complete atrophy (Fig. 2A).

In summary, with the exception of one dog of the highdose group (Animal ID Z592), most of the clinical ophthalmic findings in the treated eyes of all four treatment groups were observed postoperatively and considered to be related to either the surgical procedure or the medication regimen. No such findings were seen in the contralateral untreated eyes.

In vivo OCT. At week 10, assessment of ONL thickness was performed on a single OCT B scan selected within the treated area of each injected eye and on the equivalent area of each contralateral uninjected eye (Supplementary Fig. S2A). Comparison of the mean ONL thickness of the injected and uninjected eyes showed no statistical difference in the vehicle-treatment group. However, the ONL was significantly thicker in the injected eyes of the low- $(p \leq 0.05)$, mid- $(p \leq 0.01)$, and the high-dose groups $(p \leq 0.001)$ (Supplementary Fig. S3A). When the mean interocular difference in ONL thickness was calculated by treatment group, and compared with the vehicle-injected group, a statistically significant rescue effect was found for the mid- $(p \leq 0.01)$ and high$(p \leq 0.05)$ dose groups (Supplementary Fig. S3B).

At week 20, none of the three vehicle-injected eyes showed any signs of ONL preservation. Eleven of 13 AGTC-501 injected eyes had obvious preservation of ONL thickness in the treated area (Fig. 3A, B and Supplementary Fig. S4) that expanded beyond the original bleb delimitation captured by fundus photography immediately postinjection. One eye in the low-dose group (Animal ID Z605) had minimal to no ONL preservation when compared with the other two eyes of the same dose group; this was most likely due to some vitreal reflux at the time of injection and rapid flattening of the bleb immediately postdosage. In the high-dose group, one eye (Animal ID Z592) had marked retinal thinning and loss of lamination in the treated area, but no alterations in the untreated areas of the same eye (Fig. 2B-D). For this reason, in the high-dose injected group, all statistical 

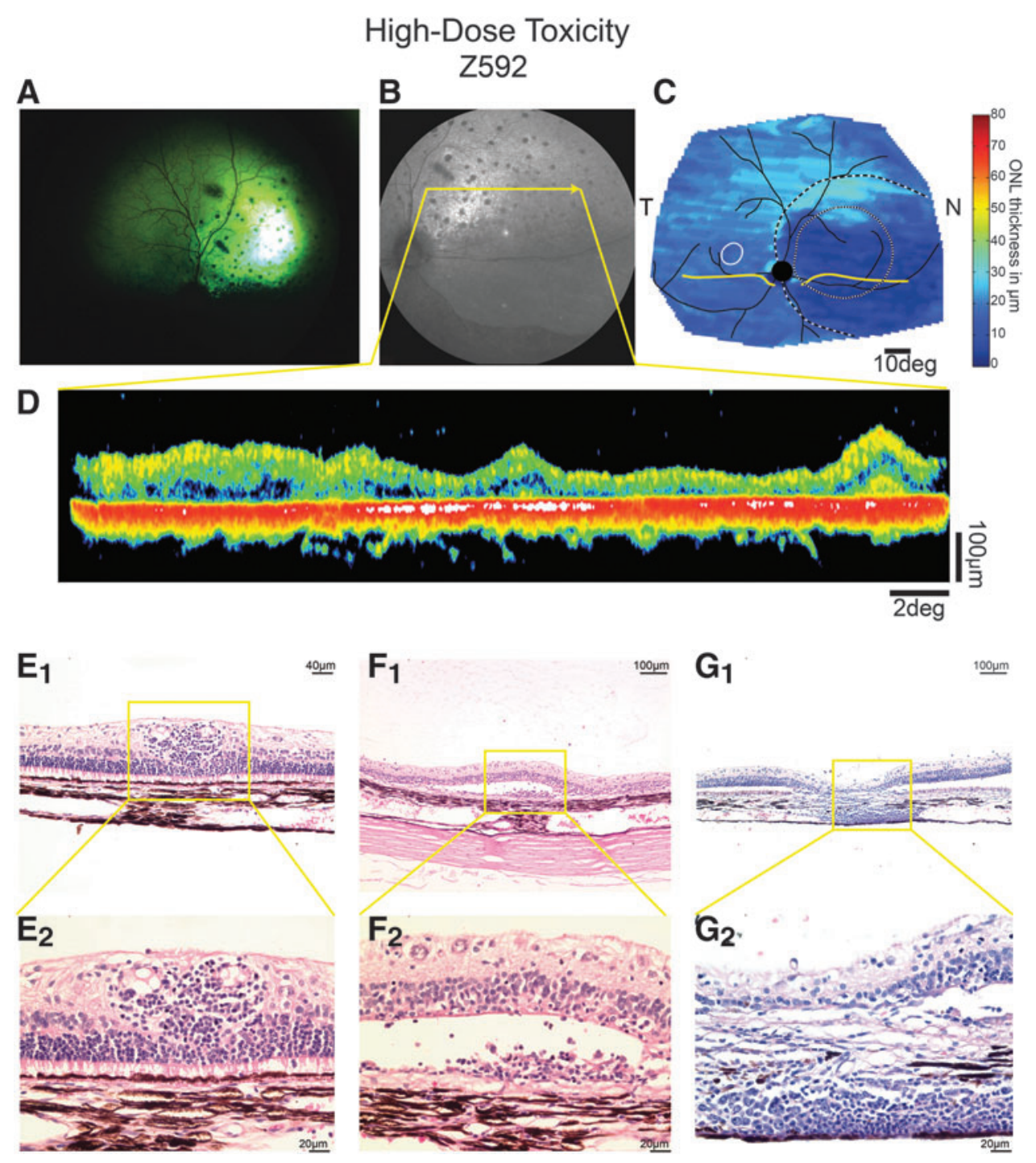

Figure 2. Clinical and histological signs of retinal toxicity in dog $Z 592$ treated with a high dose $\left(3.0 \times 10^{12} \mathrm{vg} / \mathrm{mL}\right)$ of AGTC-501. (A) Fundus photograph at 20 weeks post-treatment shows pronounced hyper-reflectivity within the treated area suggestive of retinal atrophy and multifocal black pigmentation suggestive of chorioretinitis. (B) Similar findings were observed at 19 weeks postdosage by infrared cSLO imaging [yellow arrow represents the location of the OCT B-Scan shown in (D)]. (C) Pseudocolor ONL thickness map shows loss of ONL thickness within the treated area. Treatment boundaries are based on fundus photographs of the bleb taken at the time of the injection (dotted lines), and, if visible, demarcations apparent on infrared imaging at the time of scanning (dashed lines). Optic nerve and major blood vessels (black), tapetum boundary (yellow), and fovea-like region (white ellipse) are overlaid for ease of comparison. (D) Single OCT B scan within the treated areas shows severe retinal atrophy. (E-G) Representative photomicrographs of the treated area of H\&E-stained retinal sections showing lymphoplasmacytic infiltration around blood vessels $\left(\mathbf{E}_{\mathbf{1}}, \mathbf{E}_{\mathbf{2}}\right)$, within the subretinal space $\left(\mathbf{F}_{\mathbf{1}}, \mathbf{F}_{2}\right)$, and in the choroid $\left(\mathbf{G}_{1}, \mathbf{G}_{\mathbf{2}}\right)$. cSLO, confocal laser ophthalmoscopy; H\&E, hematoxylin and eosin; N, nasal retina; OCT, optical coherence tomography; T, temporal.

analyses of ONL thickness described below were performed by both including and excluding this animal.

A more robust and extensive quantitative analysis of ONL thickness was conducted as described below and illustrated in Supplementary Figs. S2B and S5. The mean difference in ONL thickness by treatment group between the treated area of the injected eye and the untreated area of the same eye (Fig. 3C and Supplementary Figs. S2B and S5) showed a significantly thicker ONL $(p \leq 0.01)$ in the low-, mid-, and high- (excluding Animal ID Z592) dose treatment groups when compared with the vehicle-treatment group. No differences were found when analyzing equivalent areas between the different uninjected eyes (Fig. 3C).

When the mean interocular difference in ONL thickness was calculated between the treated/bleb area of the injected eye and a bleb-equivalent area of the uninjected contralateral eye (Supplementary Fig. S2B), and compared with the vehicle-injected group, a significant rescue effect was found for the low- $(p \leq 0.01)$, mid$(p \leq 0.001)$, and high- (excluding Animal ID Z592) $(p \leq 0.01)$ dose treatment groups (Fig. 3D). The ONL retention in the high-dose group was approximately twofold higher when the animal (Z592) with complete 
A Vehicle
Z587

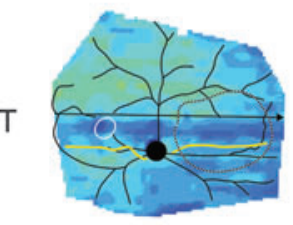

Low Dose Z558

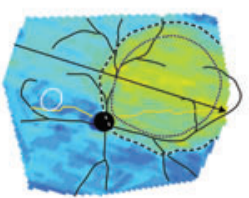

B

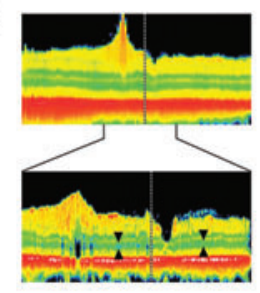

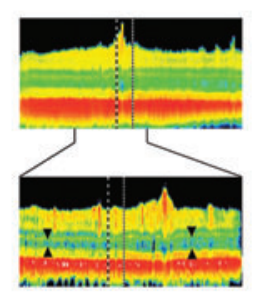

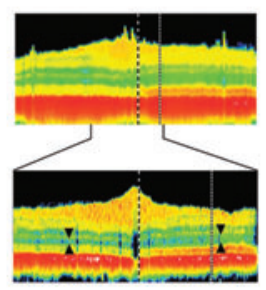

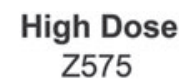

Z594
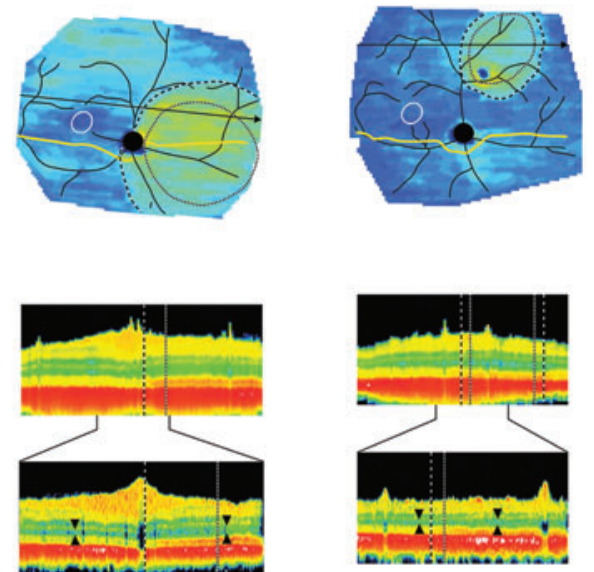

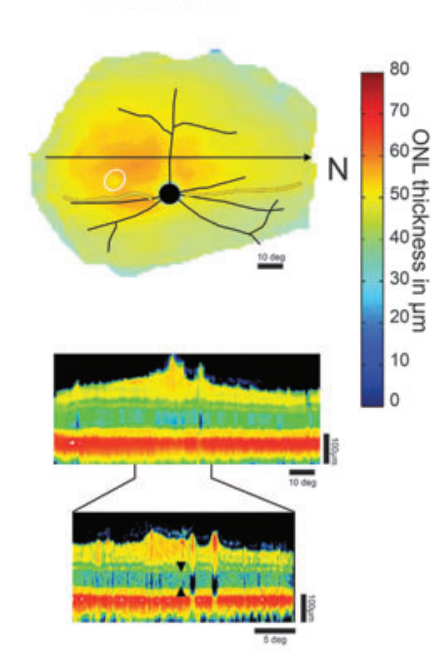

WT control
C

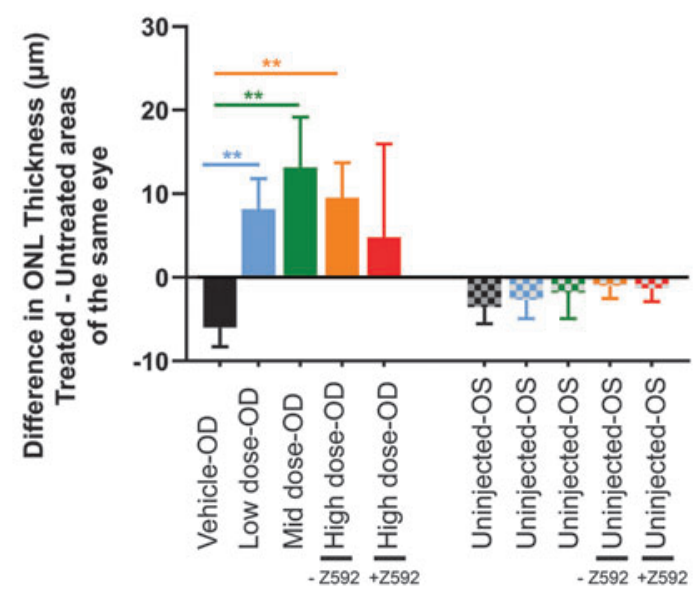

D

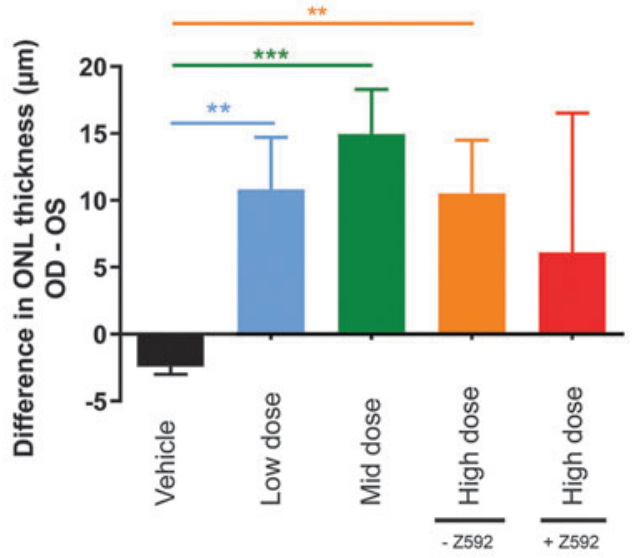

Figure 3. In vivo assessment of ONL thickness at 19-20 weeks post-treatment illustrating the level of photoreceptor protection in one dog from each treatment group. (A) Pseudocolor ONL thickness map of one dog from the vehicle-injected group, AGTC-501 low dose $\left(1.2 \times 10^{11} \mathrm{vg} / \mathrm{mL}\right), \mathrm{mid} \mathrm{dose}\left(6.0 \times 10^{11} \mathrm{vg} /\right.$ $\mathrm{mL})$, and high dose $\left(3.0 \times 10^{12} \mathrm{vg} / \mathrm{mL}\right)$. Average maps from wild-type dogs are shown for comparison. Treatment boundaries are based on fundus photographs of the bleb taken at the time of the injection (dotted lines), and, if visible, demarcations apparent on infrared imaging at the time of scanning (dashed lines). Optic nerve and major blood vessels (black), tapetum boundary (yellow), and fovea-like region (white ellipse) are overlaid for ease of comparison. Black arrow. location of OCT scan shown in (B). (B) Reconstituted horizontal OCT B-scan spanning through the treated and untreated retinal areas (upper) and individual B-scans across the treatment transition (lower). Black wedges delimit ONL within the treated and the untreated region. (C) Mean ( \pm SD) difference in ONL thickness in the bleb/treated area versus the untreated area outside of the bleb. In uninjected (OS) eyes, measurements were taken at equivalent treated and untreated areas. (D) Comparison across treatment groups of the difference (OD-OS) in ONL thickness between the bleb/treated areas in injected eyes (OD) and bleb-equivalent area of contralateral uninjected eye. Statistical analysis compared AGTC-501 treatment groups with vehicle-control group. Statistics performed with (+Z592) and without (-Z592) inclusion of the eye with toxicity. ${ }^{* *} p \leq 0.01$, ${ }^{* *} p \leq 0.001 . \mathrm{N}$, nasal retina; OD, right eye; OS, left eye; SD, standard deviation; T, temporal.

retinal atrophy was excluded, but it was comparable with the low-dose group (Fig. 3D).

Electroretinography. ERG waveforms from one representative animal of each dose group are shown in Supplementary Fig. S6. Qualitative analysis of the vehicle-treated group at both study weeks 10 and 19-20 showed no differences in the waveforms for any of the responses between the injected and contralateral uninjected eyes (Supplementary Fig. S6A, B). The low-, mid-, and high-dose groups showed better preserved waveforms in the treated (OD) eyes for the rod and, to a lesser extent, for the mixed rod-cone responses at both study weeks 10 and 19-20. Differences in cone ERG waveforms in favor of the treated eye were observed (e.g., Animal ID Z575-Supplementary Fig. S6A, B) in some but not all animals. These results are consistent with the natural history of the disease in this model where rod function loss precedes and progresses faster than that of cones. $^{18}$

At both weeks 10 and 19-20, the difference in mean ERG amplitudes between treated (OD) and the untreated (OS) eyes was higher in the three AGTC-501 treatment groups when compared with the vehicle-treated group 
A

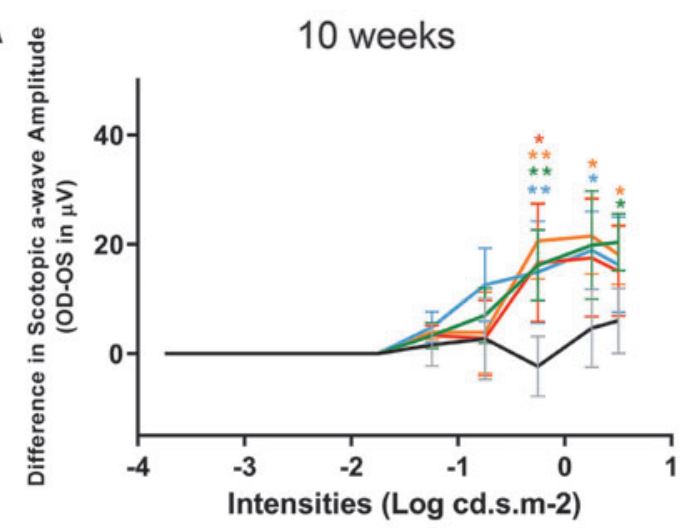

C

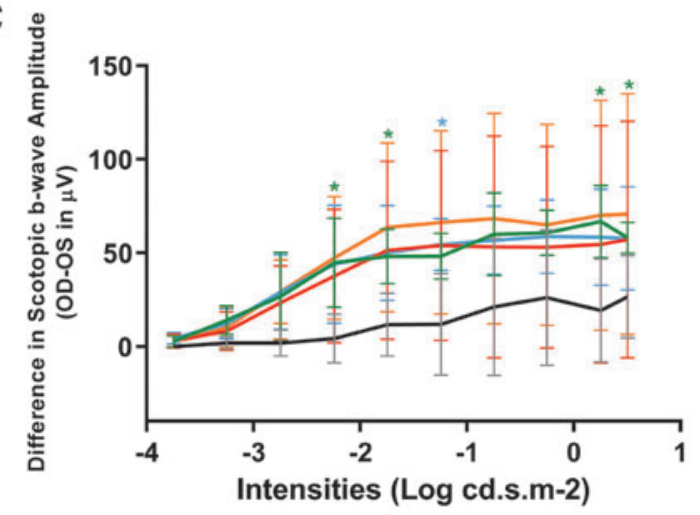

E

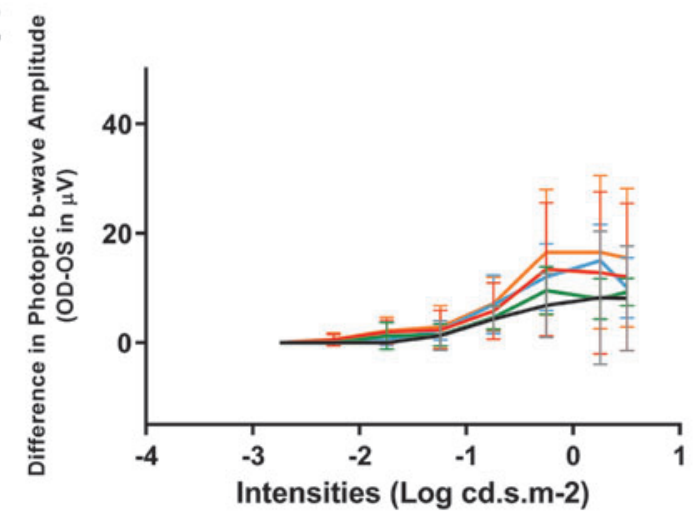

G

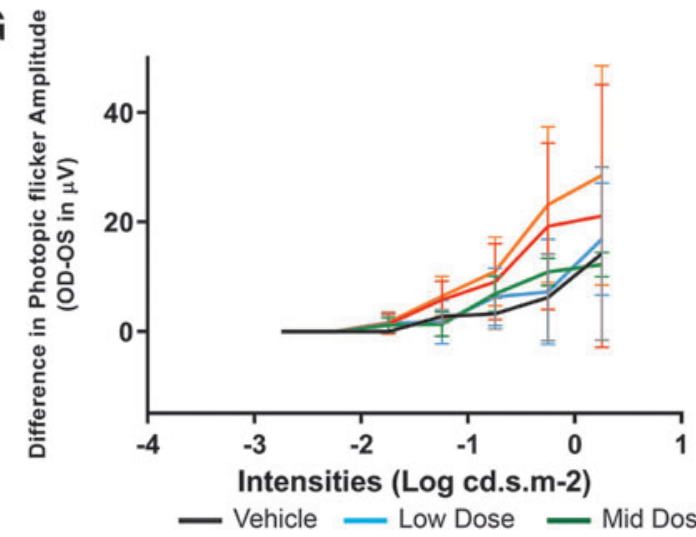

B

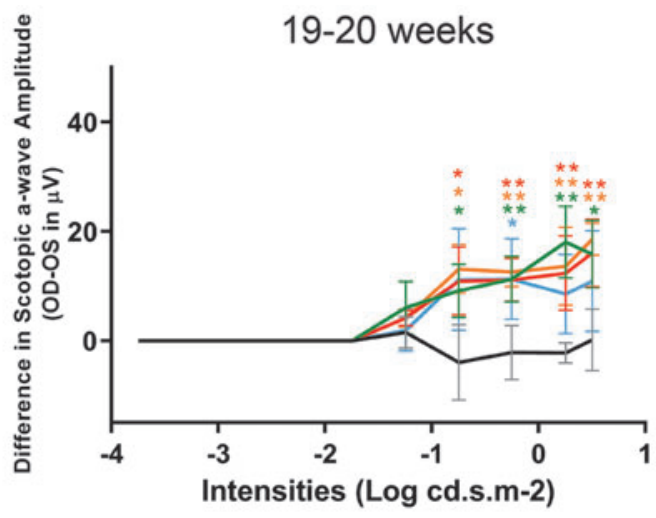

D

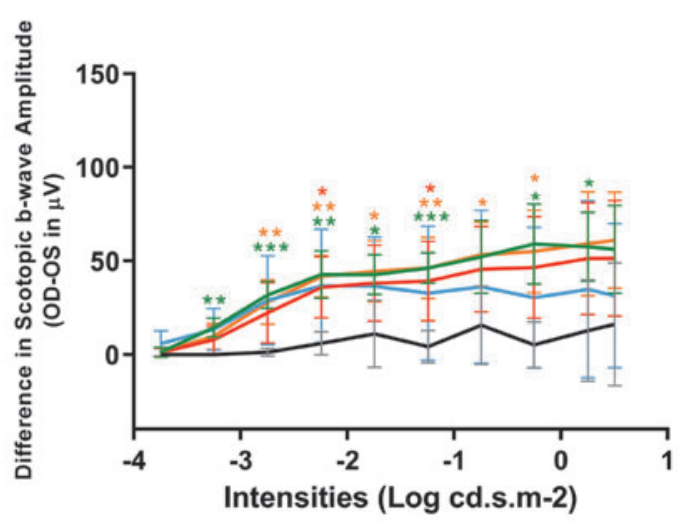

F

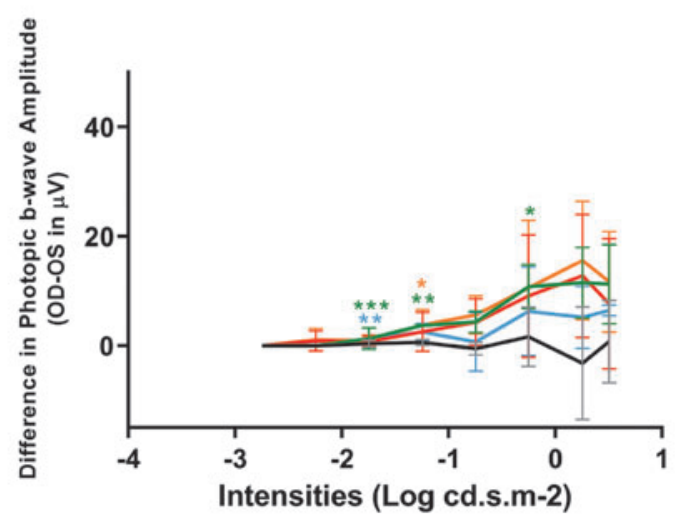

H

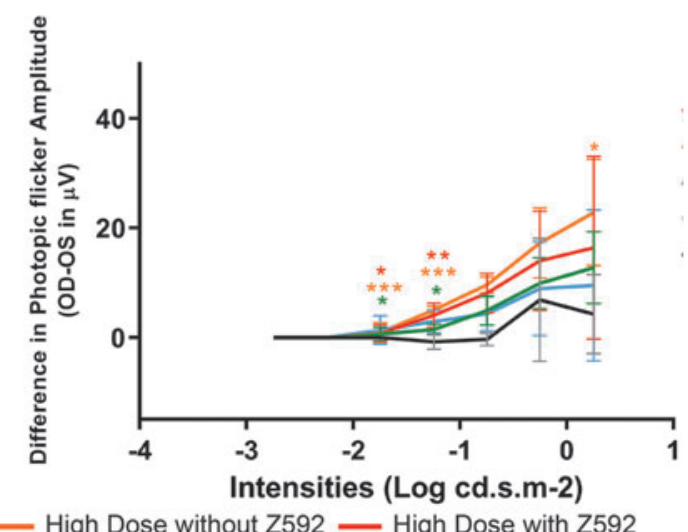

Figure 4. Differences (Mean $\pm S D$ ) in electroretinographic amplitudes between injected right eyes (OD) and uninjected left eyes (OS) from all four treatment groups at 10 and 19-20 weeks post-treatment. Differences in scotopic a-wave (A, B), scotopic b-wave (C, D), photopic b-wave (E, F), and photopic 29 Hz flicker (G, H) amplitudes are shown as a function of full-field flash intensity. ${ }^{*} p \leq 0.05,{ }^{* *} p \leq 0.01,{ }^{* * *} p \leq 0.001$. 
(Fig. 4). The most robust outcome measure of a treatment effect detectable as early as week 10 was the rod and mixed rod-cone scotopic ERG amplitude under all light stimulus conditions (Fig. 4A-D). For photopic cone ERG at 1 and $29 \mathrm{~Hz}$ stimulus conditions, a treatment effect was detectable at week 10 (Fig. 4E, G). At week 20, the mean amplitude differences between the treated groups and the vehicle were significant at the lower stimulus intensities (Fig. 4F, H).

Clinical pathology. There were no test-articlerelated effects on hematology, coagulation, or clinical chemistry parameters in any of the treatment groups. The observed changes were considered to be related to concurrent medication such as corticosteroids administered postdosage.

Nonocular histopathology. There were no testarticle-related effects on organ weights. None of the gross pathology and histopathological findings were test article related, but one dog of the high-dose group (Animal ID Z592) had a focal mononuclear infiltrate along the optic tract of the injected eye. The same animal presented a severe chorioretinitis and retinal atrophy (see below). Gross pathology and histopathological findings are summarized in Supplementary Tables S5 and S6.

Ocular histopathology. All findings are summarized in Supplementary Table S7. None of the lesions found in the vehicle-, low-, and mid-dose injected eyes were suspected to be test article related. In the high-dose injected group, five of five eyes had mild-to-moderate perivascular infiltrates of small lymphocytes and plasma cells within the ganglion cell layer and the optic nerve head (Supplementary Fig. S7). The infiltration within the ganglion cell layer was primarily seen in the superonasal quadrant, which corresponds to the location of the subretinal bleb. In one eye, these cell infiltrates expanded into the inner retina. Similar mononuclear cell infiltrates of minimal intensity were seen in all animals in the optic disk of nonhuman primates (NHPs) used in a nonclinical study for achromatopsia with the same AAV2tYF vector at a concentration of $4 \times 10^{11}$ or $4 \times 10^{12} \mathrm{vg} / \mathrm{mL}$ (corresponding to a dose of $1.2 \times 10^{11}$ and $1.2 \times 10^{12} \mathrm{vg} /$ eye $) .{ }^{26}$ Retinal lymphocytic perivascular infiltration was also seen in sheep treated for another form of achromatopsia with AAV2tYF at a concentration of $3 \times 10^{12} \mathrm{vg} / \mathrm{mL}$ (corresponding to a dose of $1.5 \times 10^{12} \mathrm{vg} /$ eye) ${ }^{27}$ It is unclear whether the cellular inflammation observed in this study is the result of an immune reaction directed against the viral capsid or the transgene. A recent study that examined the ocular inflammatory response in NHPs intravitreally injected with an AAV2tYF vector carrying a GFP reporter gene under control of a ubiquitous cytomegalovirus promoter suggests that both the capsid proteins and the viral genome contribute to posterior segment inflammation. ${ }^{38}$ Improving vector manufacturing technologies that enable reduction of the total viral load by removing empty or partially full (ineffective) capsids, while providing the same therapeutic effect, is likely to help reduce AAVassociated inflammation. While the most likely cause for the toxicity observed in the high-dose group is the result of an immune reaction directed against the virus (capsid and/ or viral genome), we cannot exclude the possibility that overproduction of the RPGR ${ }^{\mathrm{ex} 1-\mathrm{ORF} 15}$ protein resulted in cytotoxicity as a consequence of protein aggregation or impaired interaction with some of its numerous binding partners. ${ }^{39}$

In the high-dose group, Animal ID Z592 had severe lesions in the treated eye that consisted of ONL loss/ complete retinal atrophy, multifocal moderate perivascular infiltrate of small lymphocytes (Fig. 2 $\mathrm{E}_{1}, \mathrm{E}_{2}$ ), few plasma cells within the ganglion cells layer and optic nerve head, and a mild similar infiltration in the inner nuclear layer, subretinal space (Fig. $2 \mathrm{~F}_{1}, \mathrm{~F}_{2}$ ) and choroid (Fig. $2 \mathrm{G}_{1}, \mathrm{G}_{2}$ ). These findings resemble that previously observed in sheep subretinally injected with a rAAV2tYFPR1.7-hCNGA3 vector at a dose of $1.5 \times 10^{12}$ vg/eye. $^{27}$ The contralateral eye of Animal ID Z592 had minimal perivascular plasmacytic infiltrates within the retinal ganglion cell layer. It was the only contralateral/uninjected eye that showed any lesions across all treatment groups.

When excluding the eye with severe retinal atrophy (Z592-OD), assessment of H\&E-stained retinal sections that included the treated area of all 12 eyes showed better photoreceptor inner and outer segment preservation in AGTC-501-treated than in vehicle-injected eyes (Fig. 5A). Spidergrams of these retinas showed greater ONL thickness in the treated area of the AGTC-501-injected eyes in the mid-dose and high-dose groups, and in two of four in the low-dose group (Fig. 5B and Supplementary Fig. S8). It is important to note that the spidergrams represent a single $6 \mu \mathrm{m}$ cross-sectional view of the retina (Supplementary Fig. S2C) and do not represent the global ONL thickness assessment performed on the OCT data at week 20 (Supplementary Fig. S2B).

Quantitative assessment of mean ONL thickness within the treated area of the injected eye compared with an equivalent area of the contralateral uninjected eye showed no differences in the vehicle-treatment group while a significantly thicker ONL was found in the treated areas of the low- $(p \leq 0.05)$, mid- $(p \leq 0.01)$, and high- $(p \leq 0.01)$ dose (excluding Z592) treatment groups (Fig. 5C). When mean interocular difference in ONL thickness was calculated and compared with that of the vehicle-injected eye, a statistically significant $(p \leq 0.01)$ rescue effect was found in the mid- and high- (excluding Z592) dose treatment groups. Although there seemed to have been a rescue effect with a mean (SD) of 6.7 (3.7) $\mu \mathrm{m}$ in the low-dose group, it was not statistically significant (Fig. 5D). 
A
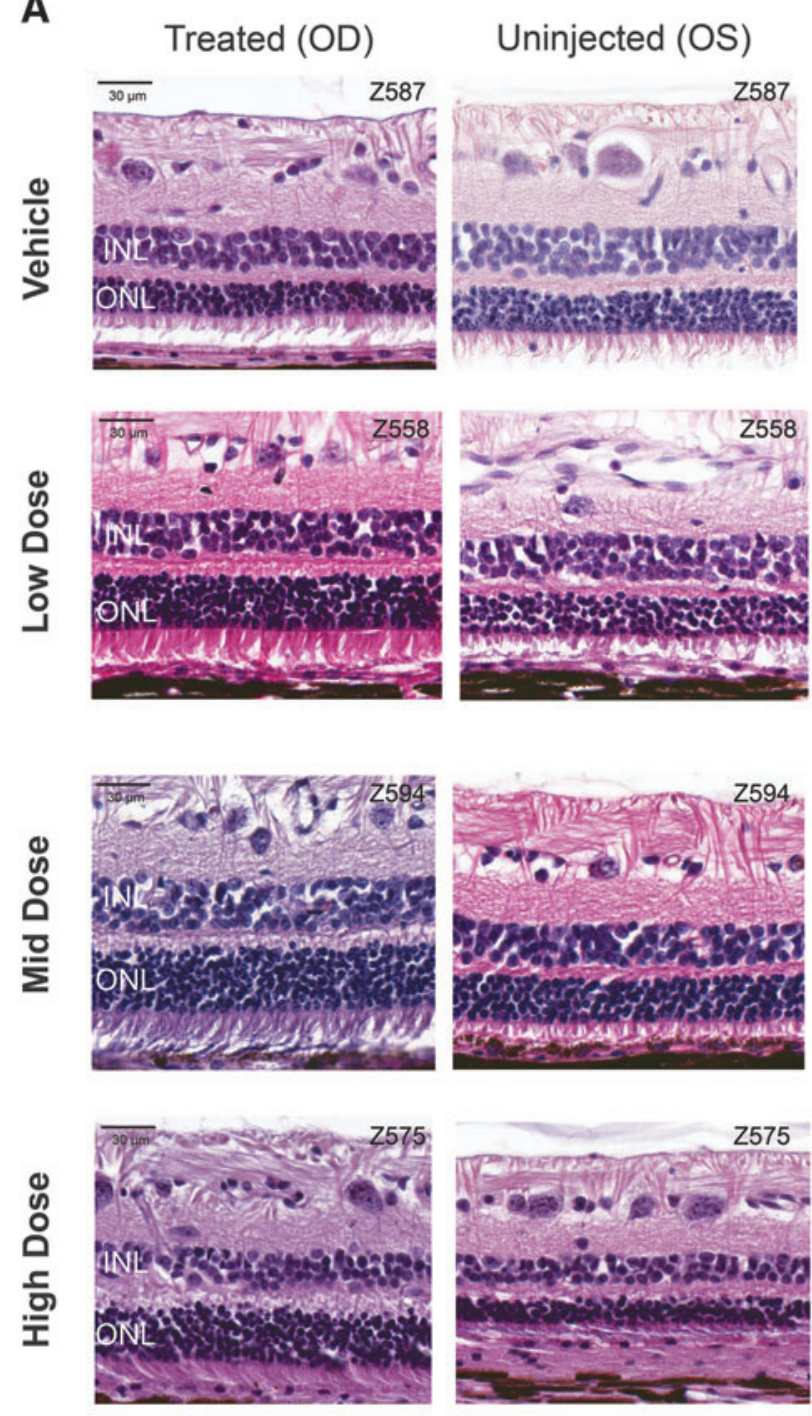

C

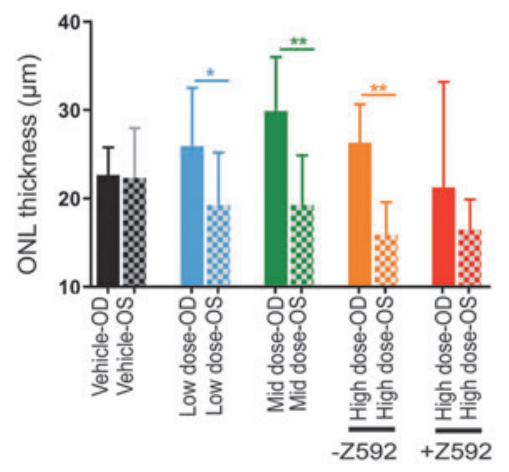

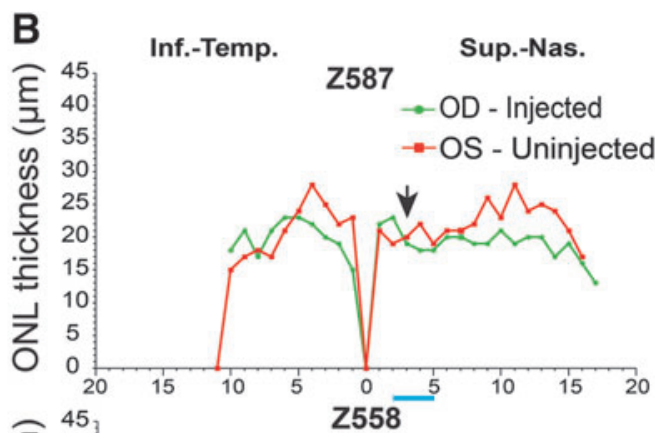
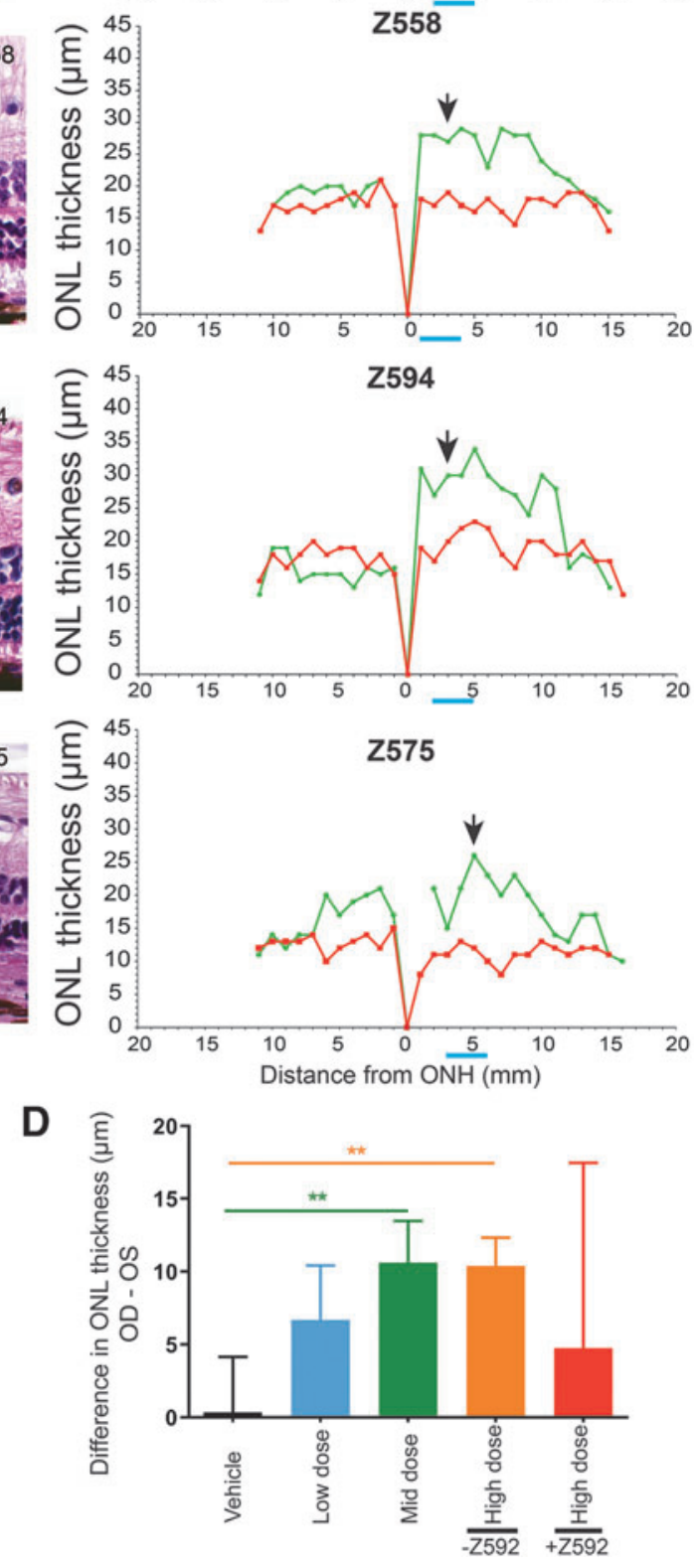

Figure 5. Representative retinal histology and quantification of ONL thickness at 20-21 weeks postdosage in treated and uninjected eyes from all four treatment groups. (A) Photomicrographs of H\&E-stained sections showing the retinal morphology in the treated area and equivalent location of the contralateral uninjected eyes [Location of section is the black arrow in (B)]. (B) Spidergraphs of ONL thickness measured in both eyes (green trace=0D/ injected right eye; red trace $=0 \mathrm{~S} /$ uninjected left eye) that extended from optic nerve head (ONH) to the peripheral ora serrata along both the Inf.-Temp. and Sup.-Nas. quadrants and encompassed the bleb and bleb-equivalent regions. ONL: outer nuclear layer; INL: inner nuclear layer. Blue bar under $\mathrm{x}$ axis of spidergraphs corresponds to four locations within the bleb/treated area (and equivalent area in OS) used for calculation of the mean ONL thickness. (C) Mean ( \pm SD) ONL thickness in injected and uninjected eyes of vehicle, low-dose, mid-dose, and high (with and without animal ID Z592)-dose groups. (D) Comparison across treatment groups of the difference (OD-OS) in ONL thickness between the injected eyes (OD) and the contralateral uninjected eyes (OS) of the vehicle, low-dose, mid-dose, and high (with and without animal ID Z592)-dose groups. Statistical analysis compared AGTC-501 treatment groups with vehicle-control group. ${ }^{*} p \leq 0.05,{ }^{* *} p \leq 0.01$. Inf.-Temp., inferotemporal; Sup.-Nas., superonasal. 


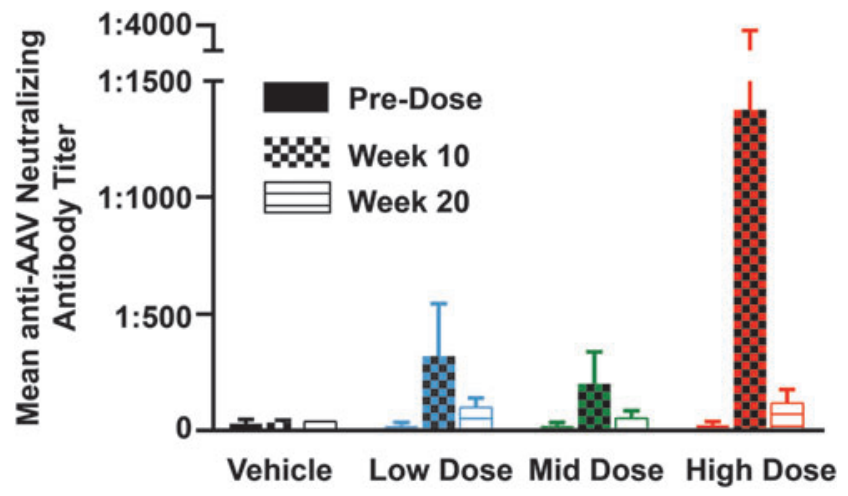

Figure 6. Serum titers of neutralizing antibodies against AAV2tYF capsid proteins. Titers (mean $\pm S D$ ) of neutralizing antibodies (expressed as inverse of greatest dilution) were measured in serum collected at baseline (predosage), 10 weeks postdosage, and at termination 20-21 weeks postdosage from all dogs of the four treatment groups.

Immunological studies. All serum samples were negative for anti-hRPGR antibodies at all time points. Mean neutralizing AAV antibody titers (expressed as inverse of greatest dilution) directed against AAV2tYF from animals of each treatment group are shown in Fig. 6. In the vehicle-treated group, variation in titers at the three time points was minor. An increase in neutralizing AAV antibody titers was seen in all three AGTC-501 treatment groups at week 10 but was reduced at week 20 . Neutralizing AAV antibody titers measured at week 10 in three of the five dogs from the high-dose group were similar to that of animals from both the low-dose and mid-dose groups. However, increased AAV antibody titer levels were detected in the remaining two dogs (Animal ID Z575, Z592) with values as high as 1:1,280 and 1:5,120, respectively, at week 10 . These two animals also showed funduscopic abnormalities characterized by dilated retinal vessels, and Z592 showed chorioretinitis as well (Supplementary Table S4) at the time of increase of neutralizing AAV antibody titers.

\section{CONCLUSIONS}

We have evaluated the toxicity and efficacy of a viral vector developed for the treatment of $R P G R$-XLRP in a naturally occurring canine model. In vivo OCT imaging and ERG analysis showed evidence of preservation of photoreceptor structure and function in the low-, mid-, and high-dose treatment groups when compared with the vehicle-treatment group. Histological analysis confirmed that retention of photoreceptor structure not associated with any side effects in the low- and mid-dose treatment groups. However, five of five animals treated with the high dose of AGTC-501 showed signs of retinal and optic nerve head inflammation in the injected eye. In one animal, there was complete retinal atrophy in the area of treatment. These results define the no-observed-adverse-effect level as $6.0 \times 10^{11} \mathrm{vg} / \mathrm{mL}$ (subretinally injected in $0.07 \mathrm{~mL}$ volume, $4.2 \times 10^{10} \mathrm{vg} /$ eye) and support the use of AGTC501 in clinical studies with patients affected with retinitis pigmentosa caused by RPGR-ORF15 mutations.

\section{ACKNOWLEDGMENTS}

The authors thank Teresa Jordan and the RDSF staff for canine husbandry and assistance in all in-life studies, Svetlana Savina and Inna Martynyuk for histotechnical assistance, Lydia Melnyk for research coordination, and Drs. Jun Xie and Guangping Gao for AAV neutralizing antibody measurement.

\section{AUTHOR DISCLOSURE}

C.S., G.Y., A.T., D.R.K., M.S., P.M.R., and J.D.C. are employees and shareholders of AGTC and have a conflict of interest to the extent that this work potentially increases their financial interests. None of the other authors have a competing financial interest.

\section{FUNDING INFORMATION}

This work was supported by a Sponsored Research Agreement from AGTC, Inc. to the University of Pennsylvania, research grants from NIH/National Eye Institute R01-EY06855, P30-EY001583, and from the Foundation Fighting Blindness.

\section{SUPPLEMENTARY MATERIAL}

Supplementary Table S1

Supplementary Table S2

Supplementary Table S3

Supplementary Table S4

Supplementary Table S5

Supplementary Table S6

Supplementary Table S7

Supplementary Figure S1

Supplementary Figure S2

Supplementary Figure S3

Supplementary Figure S4

Supplementary Figure S5

Supplementary Figure S6

Supplementary Figure S7

Supplementary Figure S8 


\section{REFERENCES}

1. Fishman GA. Retinitis pigmentosa. Genetic percentages. Arch Ophthalmol 1978;96:822-826.

2. Jay M. On the heredity of retinitis pigmentosa. $\mathrm{Br}$ J Ophthalmol 1982;66:405-416.

3. Breuer DK, Yashar BM, Filippova E, et al. A comprehensive mutation analysis of RP2 and RPGR in a North American cohort of families with $X$-linked retinitis pigmentosa. Am J Hum Genet 2002;70:1545-1554.

4. Bader I, Brandau O, Achatz $\mathrm{H}$, et al. X-linked retinitis pigmentosa: RPGR mutations in most families with definite $X$ linkage and clustering of mutations in a short sequence stretch of exon ORF15. Invest Ophthalmol Vis Sci 2003:44:14581463

5. Sharon D, Sandberg MA, Rabe VW, et al. RP2 and RPGR mutations and clinical correlations in patients with $\mathrm{X}$-linked retinitis pigmentosa. Am J Hum Genet 2003;73:1131-1146.

6. Pelletier V, Jambou M, Delphin N, et al. Comprehensive survey of mutations in RP2 and RPGR in patients affected with distinct retinal dystrophies: genotype-phenotype correlations and impact on genetic counseling. Hum Mutat 2007;28: 81-91.

7. Branham K, Othman M, Brumm M, et al. Mutations in RPGR and RP2 account for $15 \%$ of males with simplex retinal degenerative disease. Invest Ophthalmol Vis Sci 2012;53:8232-8237.

8. Shu X, Black GC, Rice JM, et al. RPGR mutation analysis and disease: an update. Hum Mutat 2007;28:322-328.

9. Charng J, Cideciyan AV, Jacobson SG, et al. Variegated yet non-random rod and cone photoreceptor disease patterns in RPGR-ORF15associated retinal degeneration. Hum Mol Genet 2016;25:5444-5459.

10. Vervoort $R$, Lennon $A$, Bird $A C$, et al. Mutational hot spot within a new RPGR exon in X-linked retinitis pigmentosa. Nat Genet 2000;25:462-466.

11. Neidhardt J, Glaus E, Barthelmes D, et al. Identification and characterization of a novel RPGR isoform in human retina. Hum Mutat 2007;28: 797-807.

12. Schmid F, Glaus E, Cremers FP, et al. Mutationand tissue-specific alterations of RPGR transcripts. Invest Ophthalmol Vis Sci 2010;51:1628-1635.

13. Hong DH, Pawlyk B, Sokolov M, et al. RPGR isoforms in photoreceptor connecting cilia and the transitional zone of motile cilia. Invest Ophthalmol Vis Sci 2003;44:2413-2421.

14. Wright RN, Hong DH, Perkins B. Misexpression of the constitutive Rpgr(ex1-19) variant leads to severe photoreceptor degeneration. Invest Ophthalmol Vis Sci 2011;52:5189-5201.

15. Rao KN, Li L, Anand M, et al. Ablation of retinal ciliopathy protein RPGR results in altered photoreceptor ciliary composition. Sci Rep 2015;5: 11137.

16. Khanna $\mathrm{H}$. More than meets the eye: current understanding of RPGR function. Adv Exp Med Biol 2018;1074:521-538.

17. Zeiss CJ, Acland GM, Aguirre GD. Retinal pathology of canine $\mathrm{X}$-linked progressive retinal atrophy, the locus homologue of RP3. Invest Ophthalmol Vis Sci 1999;40:3292-3304.

18. Zhang 0 , Acland GM, Wu WX, et al. Different RPGR exon ORF15 mutations in Canids provide insights into photoreceptor cell degeneration. Hum Mol Genet 2002;11:993-1003.

19. Beltran WA, Hammond P, Acland GM, et al. A frameshift mutation in RPGR exon ORF15 causes photoreceptor degeneration and inner retina remodeling in a model of $X$-linked retinitis pigmentosa. Invest Ophthalmol Vis Sci 2006;47: 1669-1681.

20. Beltran WA, Cideciyan AV, Lewin AS, et al. Gene therapy rescues $X$-linked photoreceptor blindness in dogs and paves the way for treating RPGR form of human retinitis pigmentosa. Proc Natl Acad Sci U S A 2012;109:2132-2137.

21. Beltran WA, Cideciyan AV, Iwabe $S$, et al. Successful arrest of photoreceptor and vision loss expands the therapeutic window of retinal gene therapy to later stages of disease. Proc Natl Acad Sci U S A 2015;112:E5844-E5853.

22. Wu Z, Hiriyanna S, Qian $H$, et al. A long-term efficacy study of gene replacement therapy for RPGR-associated retinal degeneration. Hum Mol Genet 2015;24:3956-3970.

23. Fischer MD, McClements ME, Martinez-Fernandez de la Camara C, et al. Codon-optimized RPGR improves stability and efficacy of AAV8 gene therapy in two mouse models of $X$-linked retinitis pigmentosa. Mol Ther 2017;25:1854-1865.

24. Beltran WA, Cideciyan AV, Boye SE, et al. Optimization of retinal gene therapy for $\mathrm{X}$-linked retinitis pigmentosa due to RPGR mutations. Mol Ther 2017;25:1866-1880.

25. Song C, Conlon TJ, Deng WT, et al. Toxicology and pharmacology of an AAV vector expressing codon-optimized RPGR in RPGR-deficient Rd9 mice. Hum Gene Ther Clin Dev 2018;29: 188-197.

26. Ye GJ, Budzynski E, Sonnentag P, et al. Safety and biodistribution evaluation in cynomolgus macaques of rAAV2tYF-PR1.7-hCNGB3, a recombinant AAV vector for treatment of achromatopsia. Hum Gene Ther Clin Dev 2016;27:37-48.

27. Gootwine E, Ofri R, Banin E, et al. Safety and efficacy evaluation of rAAV2tYF-PR1.7-hCNGA3 vector delivered by subretinal injection in CNGA3 mutant achromatopsia sheep. Hum Gene Ther Clin Dev 2017;28:96-107.
28. Kang W, Wang L, Harrell $H$, et al. An efficient rHSV-based complementation system for the production of multiple rAAV vector serotypes. Gene Ther 2009;16:229-239.

29. Thomas DL, Wang L, Niamke J, et al. Scalable recombinant adeno-associated virus production using recombinant herpes simplex virus type 1 coinfection of suspension-adapted mammalian cells. Hum Gene Ther 2009;20:861-870.

30. Ye GJ, Budzynski E, Sonnentag P, et al. Safety and biodistribution evaluation in cynomolgus macaques of rAAV2tYF-CB-hRS1, a recombinant adeno-associated virus vector expressing retinoschisin. Hum Gene Ther Clin Dev 2015;26: 165-176.

31. Chulay JD, Ye GJ, Thomas DL, et al. Preclinical evaluation of a recombinant adeno-associated virus vector expressing human alpha-1 antitrypsin made using a recombinant herpes simplex virus production method. Hum Gene Ther 2011;22: 155-165.

32. Komaromy AM, Varner SE, de Juan E, et al. Application of a new subretinal injection device in the dog. Cell Transplant 2006;15:511-519.

33. Beltran WA, Boye SL, Boye SE, et al. rAAV2/5 gene-targeting to rods:dose-dependent efficiency and complications associated with different promoters. Gene Ther 2010;17:1162-1174.

34. Calcedo R, Vandenberghe LH, Gao G, et al Worldwide epidemiology of neutralizing antibodies to adeno-associated viruses. J Infect Dis 2009;199:381-390.

35. Iwabe S, Ying GS, Aguirre GD, et al. Assessment of visual function and retinal structure following acute light exposure in the light sensitive T4R rhodopsin mutant dog. Exp Eye Res 2016;146: 341-353.

36. Edelmann ML, Miyadera K, Iwabe S, et al. Investigating the inheritance of prolapsed nictitating membrane glands in a large canine pedigree. Vet Ophthalmol 2013;16:416-422.

37. Ribas Latre A, McPartland A, Cain D, et al. Canine sterile steroid-responsive lymphadenitis in 49 dogs. J Small Anim Pract 2019;60:280-290.

38. Timmers AM, Newmark JA, Turunen HT, et al. Ocular inflammatory response to intravitreal injection of adeno-associated virus vector: relative contribution of genome and capsid. Hum Gene Ther 2020;31:80-89.

39. Megaw RD, Soares DC, Wright AF. RPGR: its role in photoreceptor physiology, human disease, and future therapies. Exp Eye Res 2015;138:32-41.

Received for publication October 13, 2019; accepted after revision December 15, 2019.

Published online: January 3, 2020. 